\title{
Les misères de Paris
}

Des représentations du corps souffrant dans la Revue photographique des hôpitaux de Paris

Miseries of Paris. Representation of the suffering bodies in the Revue

photographique des hôpitaux de Paris

\section{Axel Hohnsbein}

\section{(2) OpenEdition \\ Journals}

Édition électronique

URL : https://journals.openedition.org/aes/4260

DOI : 10.4000/aes.4260

ISSN : 2258-093X

Éditeur

Laboratoire LISAA

Référence électronique

Axel Hohnsbein, «Les misères de Paris », Arts et Savoirs [En ligne], 16 | 2021, mis en ligne le 17

décembre 2021, consulté le 19 décembre 2021. URL : http://journals.openedition.org/aes/4260 ; DOI : https://doi.org/10.4000/aes.4260

Ce document a été généré automatiquement le 19 décembre 2021.

Centre de recherche LISAA (Littératures SAvoirs et Arts) 


\title{
Les misères de Paris
}

\author{
Des représentations du corps souffrant dans la Revue photographique \\ des hôpitaux de Paris
}

\author{
Miseries of Paris. Representation of the suffering bodies in the Revue \\ photographique des hôpitaux de Paris
}

\section{Axel Hohnsbein}

1 1867: les premières livraisons de la Clinique photographique de l'hôpital Saint-Louis commencent à paraître. Dirigée par Alfred Hardy, professeur de pathologie spécialisé dans la dermatologie, et son élève Arthur de Montméja, qui s'est formé à la photographie pour l'occasion, cette œuvre se présente comme « un recueil destiné à faciliter l'étude des maladies de la peau, aussi bien pour les étudiants qui veulent les apprendre que pour les médecins qui éprouvent le besoin de les revoir et de se familiariser avec certains détails de ces affections ${ }^{1}$. Il s'agit d'un ouvrage pionnier, inspiré selon Arthur de Montméja par des travaux anglais qu'Alfred Hardy aurait consultés durant l'été $1866^{2}$. En France, la photographie médicale - au sens large s'illustre alors principalement par les travaux de Duchenne de Boulogne (Mécanisme de la physionomie humaine, 1862) et les photographies de l'hermaphrodite réalisées par Nadar en $1860^{3}$. Le physiologiste Étienne-Jules Marey, qui se spécialise dans l'étude du mouvement, travaille à établir sa méthode graphique et n'envisage pas encore d'utiliser la photographie.

21869 : Montméja s'associe au médecin et vulgarisateur Jules Rengade pour lancer la Revue photographique des hôpitaux de Paris. La visée n'est plus la même : abandonnant la spécialité dermatologique, renonçant à la démarche propre aux œuvres individuelles, Montméja et Rengade souhaitent «offrir au public médical [...] les cas les plus intéressants recueillis dans les hôpitaux de Paris " $^{4}$. C'est, à proprement parler, l'entrée de la photographie médicale dans le régime médiatique. Si Duchenne de Boulogne et Hardy se donnaient pour tâche de réaliser un « atlas $»^{5}$ des expressions physionomiques ou des pathologies dermatologiques, Montméja et Rengade se lancent dans la publication de cas exclusivement retenus parce qu'ils sont « les plus intéressants »- la polysémie du mot n'étant jamais résolue : il ne s'agit plus de l'étude du corps humain en 
général, mais des divers corps qui nous entourent, et plus particulièrement des corps vus dans les hôpitaux de Paris.

3 Notre objectif sera double: montrer qu'en passant de l'atlas à une étude de cas photographiés au fil de l'eau, cette revue transforme de facto des individus malades en objets médiatiques, ce qui pose la question des modalités de l'exhibition médicale; étudier la façon dont la revue orchestre la médiatisation conjointe des cas pathologiques et des institutions hospitalières de Paris. Nous commencerons par montrer que cette entreprise de centralisation médiatique des représentations du corps souffrant s'inscrit dans la démarche, courante à l'ère des Expositions universelles, de valorisation de la modernité parisienne - les cas pathologiques étant pour ainsi dire appelés à devenir des objets de fierté hospitalière ; puis nous étudierons le regard que pose la revue sur ces corps : tout en établissant la sociologie en creux d'un certain Paris, la revue cherche à décrire des affections le plus souvent sélectionnées pour leur caractère spectaculaire et permettant de présenter des images aussi extrêmes que possible; enfin, nous montrerons que ce type de photographie médicale ne parviendra pas à s'implanter : la capitalisation médiatique opérée sur ces corps se soldera par un échec, favorisant l'orientation de la photographie vers d'autres champs d'études pathologiques.

\section{Médiatiser les cas pathologiques rares de Paris}

Parue en 1868 en librairie, la Clinique photographique de l'hôpital Saint-Louis est d'emblée porteuse d'ambiguïté. Initialement publiée sous forme de quatorze fascicules ayant vocation à former un livre ${ }^{6}$, cette œuvre est précédée de deux avant-propos très différents. Alfred Hardy conçoit effectivement ce livre comme un « atlas exact pouvant former le complément de tout ouvrage de dermatologie, quelle que soit la doctrine de l'auteur $~$. Jouant pleinement son rôle de spécialiste en dermatologie, il se déleste d'une " partie artistique » qui, si elle est " sans contredit la plus importante ", n'en est pas moins "confiée à un de [s]es élèves, $M$. de Montméja ${ }^{8}$. Ce dernier se montre beaucoup moins attaché à l'ethos du médecin: laissant de côté son statut d'ancien interne, il revendique volontiers son rôle de factotum dans la seconde partie de la préface, expliquant avoir "commenc[é] par devenir photographe", avant de se "placer [lui]-même ${ }^{9}$ à la tête de l'atelier photographique alors en train de s'ériger à l'hôpital Saint-Louis. Surtout, Montméja explique avoir entrepris ce travail « uniquement dans le but» de "rappeler » à son mentor "les cas intéressants qui semblent se donner rendez-vous dans [son] service de l'hôpital Saint-Louis $»^{10}$. Le style et l'intention ne sont pas les mêmes: alors qu'Alfred Hardy pense présenter une «collection à peu près complète" des "principales maladies qu'on rencontre à l'hôpital Saint-Louis, pendant l'espace de plusieurs mois " ${ }^{11}$, Montméja individualise chaque patient en invitant Hardy à considérer ces images comme des souvenirs. Incidemment, il dépeint l'hôpital comme un haut-lieu de l'aristocratie pathologique, où les «cas intéressants" ne sont plus contraints de venir mais se «donnent rendezvous ». Si Hardy reste dans son rôle de caution scientifique, Montméja privilégie la mondanité et la sélectivité, tant du côté du lectorat que des cas pathologiques qu'il photographie.

5 Si l'on en croit l'avant-propos de la Revue photographique des hôpitaux de Paris, "les avantages de la photographie appliquée à la médecine ont valu un plein succès à la 
clinique photographique des maladies de la peau ${{ }^{12}}^{12}$ : mettant à profit sa " plus grande expérience $»^{13}$, Montméja fait donc le choix de se lancer dans le milieu hautement compétitif ${ }^{14}$ des périodiques visant le public médical. Nombre de ces publications veulent rassembler l'ensemble des savoirs anciens et actuels et favoriser les échanges au sein de la communauté médicale de Paris, de la province et parfois de l'étranger. C'est le rôle que joue par exemple L'Abeille médicale (1844-1899). Le milieu hospitalier lui-même a ses propres périodiques ${ }^{15}$ et les sociétés savantes sont nombreuses à publier leur bulletin ${ }^{16}$. L'enjeu est dès lors de se distinguer efficacement. L'avant-propos annonce que seront publiés, dans chaque numéro, « un ou plusieurs articles des maîtres les plus estimés de la science $»^{17}$. La formule est intéressante car elle est plus volontiers employée dans la presse de vulgarisation scientifique ${ }^{18}$, ce qui est peut-être le seul élément rappelant que la Revue photographique des hôpitaux de Paris est initialement codirigée par Montméja et Jules Rengade, médecin de formation œuvrant surtout dans le cadre d'une vulgarisation grand public ${ }^{19}$. En tant que médecin, son apport est d'un intérêt limité mais l'effet publicitaire d'une telle association est incontestable.

Outre la présence des «maîtres » de la science, la revue annonce qu'on trouvera dans ses pages « les observations des cas représentés dans les planches, un compte rendu des séances de l'Académie de médecine et des Sociétés savantes, une revue des principaux journaux et des analyses bibliographiques. $»^{20}$ Cette feuille de route est comparable à celle de la concurrence, à ceci près que leur publication est le plus souvent hebdomadaire tandis que la Revue photographique est mensuelle. La façon d'aborder les petites rubriques en est changée: plus synthétiques, elles sont moins ouvertes à l'actualité immédiate et le choix frappant est fait de ne pas publier de correspondance : les voix du lecteur n'existent pas $^{21}$, la revue mettant définitivement l'accent sur l'univers clos des corps malades mis en vitrine par la photographie. L'importance de la photographie, signalée dans le titre, constitue le principal argument de vente et justifie à elle seule le tarif très élevé de 2 francs par livraison ${ }^{22}$. La revue cherche par ailleurs à tirer parti de cet avantage iconographique pour supplanter ses concurrents directs en faisant office de revue officielle de l'institution hospitalière :

M. Le Directeur général de l'Assistance publique [Armand Husson] a bien voulu placer sous son patronage la nouvelle publication, et faire construire à l'hôpital Saint-Louis un magnifique atelier de photographie, qui est le rendez-vous de ce que la pathologie a de plus intéressant et de plus rare. ${ }^{23}$

7 Le lieu de centralisation et le rang d'action ne sont plus les mêmes qu'à l'époque de la Clinique photographique $e^{24}$ : ce n'est plus le service dermatologique de Hardy qui concentre les cas intéressants du seul hôpital Saint-Louis, mais l'atelier photographique de Montméja qui attire toutes sortes de cas en provenance de tous les hôpitaux de Paris. Le style mondain ne relève plus de l'artifice rhétorique mais de la stratégie médiatique. Lorsque Montméja écrit que son « magnifique atelier de photographie » est "le rendez-vous de ce que la pathologie a de plus intéressant et de plus rare ${ }^{25}$, il reprend des expressions déjà employées dans la préface de la Clinique photographique et les associe à une pratique parisienne synonyme de pratique d'élite: chapeautée par l'Assistance publique, rédigée par les «maitres de la science ", illustrée par un procédé nouveau, sa revue s'inscrit ouvertement dans le processus de modernisation des représentations de Paris qui, sous Napoléon III, devient une ville de progrès et d'innovation. ${ }^{26}$

8 Il n'est dès lors pas étonnant de constater que, dans cet avant-propos spécifique, la rareté constitue le principal point commun de ces divers cas pathologiques: en 
renonçant à la spécialisation dermatologique pour couvrir l'ensemble des manifestations anatomo-pathologiques, en renonçant au livre pour trancher en faveur du périodique, Montméja a opté pour une plus grande hétérogénéité disciplinaire qui ne peut trouver sa résolution que dans le choix constant de cas rares. L'époque tout entière invite à opérer ce choix éditorial. Lorsqu'il publie Les Merveilles de l'Exposition universelle de 1867, Jules Mesnard écrit que "jamais, en effet, tant de productions inconnues, rares ou parfaites n'ont convergé de points aussi nombreux et aussi distants vers un même centre. ${ }^{27} \mathrm{Abondance}$, rareté et perfection : c'est exactement le propos de Montméja et Rengade, à ceci près qu'il s'agit ici de "merveilles" pathologiques. Succursale médicale des Expositions universelles, outil de centralisation pathologique, la Revue photographique devient à la fois vitrine de la modernité parisienne, des pratiques du corps médical parisien, mais aussi et surtout des corps souffrants à Paris : corps de Parisiens, corps de provinciaux venus à Paris et cas tératologiques de tous horizons. Dès lors, ce n'est plus l'élite des auteurs qui importe en premier lieu, mais la mise en exploitation médiatique d'un espace hospitalier traditionnellement inaccessible aux regards extérieurs.

\section{Corps souffrant, corps soignant : une sociologie en creux}

La Clinique photographique de l'hôpital Saint-Louis est un atlas dans lequel les patients servent de support anonyme aux textes décrivant des maladies d'un point de vue général. La Revue photographique des hôpitaux de Paris, elle, traite de patients spécifiques : le rédacteur, qui peut être soit un médecin (discours direct), soit un interne (discours indirect le plus souvent), rend généralement compte de détails intimes de la vie des patients. Olivier Faure souligne que, s'ils sont "ouverts aux seuls indigents, les hôpitaux passent avec eux un contrat tacite selon lequel "le malheureux qui vient réclamer des secours paie à la médecine ce qu'il doit à la charité" ". ${ }^{28}$ L'effet de ce «contrat que passent richesse et pauvreté dans l'organisation de l'expérience clinique ${ }^{29}$ est instantanément perceptible : aucun protocole précis n'existe encore pour protéger l'anonymat des malades et la retranscription de la phase d'anamnèse ${ }^{30}$ n'est pas homogène. Certains rédacteurs écrivent en toutes lettres le nom et le prénom des malades, d'autres sélectionnent le prénom ou donnent des initiales; les photographies cadrent souvent très large, dévoilant le visage où des parties du corps non affectées (les parties génitales sont souvent dévoilées sans motif particulier). Associées à l'âge, au sexe, au tempérament et à la profession du malade, ces informations cumulées valorisent l'idée que, une fois entré à l'hôpital, la personne ne s'appartient plus.

10 Ainsi médiatisée, l'anamnèse donne lieu à une étude sociologique involontaire et fragmentaire. Toute une variété de métiers est évoquée : un tourneur en cuivre ${ }^{31}$, un charpentier ${ }^{32}$, un ajusteur ${ }^{33}$, une couturière ${ }^{34}$, une courtière en bijoux ${ }^{35}$, un savetier ${ }^{36}$, une piqueuse de bottines ${ }^{37}$, un maraîcher ${ }^{38}$, un boulanger ${ }^{39}$, un soldat devenu garçon de magasin $^{40}$, une déménageuse ${ }^{41}$, un curé ${ }^{42}$, une ouvrière en chapellerie ${ }^{43}$, etc. Parfois, les patients se déplacent spécialement de province pour consulter, tel cet homme originaire de Champagne "venu à Paris en 1869 pour y chercher la guérison à la difformité dont il était atteint $»^{44}$; ou cet avocat d'Angers qui souhaite faire opérer son fils de quatre mois «atteint d'un bec-de-lièvre double " $^{45}$. L'anamnèse laisse souvent 
entr'apercevoir l'histoire d'une vie : ici, on étudie le cas d'une femme de soixante-neuf ans qui " a perdu sa petite fille, à laquelle elle était fort attachée », et dont l'« état de malaise s'est aggravé $»^{46}$; là, on s'intéresse à un homme, ancien tailleur de pierre, devenu marchand des quatre-saisons à cinquante-cinq ans, qui «se trouvait pendant douze à quinze heures debout, souvent à l'humidité, sans cependant en éprouver d'inconvénient ", et dont les jambes se sont un jour couvertes de plaies et de croûtes ${ }^{47}$. Les accidentés sont nombreux : ce peut être un jeune homme qui « a probablement été assommé au sortir d'un bal » ${ }^{48}$ et que l'on a "ramassé sans connaissance rue de Ravignan $»^{49}$; ou un homme de soixante-seize ans, marchand de quatre saisons, qui «a été atteint d'un coup de feu à la jambe gauche au moment où il maraudait dans les champs de Bondy $\aleph^{50}$. Parfois, une simple date raconte une histoire: une femme de trente ans, "d'une bonne santé habituelle, vivant dans d'excellentes conditions hygiéniques, mère de trois enfants bien portants, s'est exposée à un refroidissement dans la nuit du 31 décembre au $1^{\mathrm{er}}$ janvier ${ }^{51}$. Ce ne sont pas des histoires complètes, mais des bribes sociologiques servant à comprendre l'histoire d'une vie à l'échelle de la biologie.

11 L'accumulation de ces bribes au sein de la revue valide l'idée foucaldienne selon laquelle « l'espace médical peut coïncider avec l'espace social, ou plutôt le traverser et le pénétrer entièrement ». Foucault précise que, dans la première moitié du XIX siècle, " on commence à concevoir une présence généralisée des médecins dont les regards croisés forment réseau et exercent en tout point de l'espace, en tout moment du temps, une surveillance constante $»^{52}$. Dans ce passage, Foucault évoque l'implantation des médecins sur l'ensemble du territoire, mais l'hôpital se situe nécessairement à l'épicentre de ce réseau : dans la revue, le corps souffrant est souvent décrit en transit, l'hôpital constituant le lieu où la maladie connaît le plus souvent son terme. Tantôt, c'est un médecin de ville ou de campagne qui adresse le malade à l'hôpital, tantôt le malade est transféré d'hôpital en hôpital, de service en service, certains rédacteurs notant précisément le numéro de la salle, le nom du directeur du service, celui de la personne présente au moment de l'accueil du malade, etc. Ces précisions sont elles aussi données comme en passant et ne sont pas soumises à un protocole rédactionnel strict ${ }^{53}$.

Un nombre de textes assez important note l'état psychologique et le comportement des patients : c'est le cas du premier malade étudié par la revue, un homme de vingt-neuf ans souffrant d'un éléphantiasis du prépuce : le malade, «honteux de sa difformité, la cachait avec soin, et il ne vint demander les secours de la chirurgie que le jour où le poids de la tumeur l'empêcha de travailler $\aleph^{54}$. Certains articles placent pour ainsi dire côte-à-côte les malades afin de comparer les manifestations de leur maladie. Une étude du Dr. Verneuil réunit deux hommes souffrant de lipômes symétriques : Le premier s'inquiète dès les premiers symptômes, "parce qu'il y voi[t] une menace à la perfection de ses formes physiques ${ }^{55}$, tandis que le second, « ne souffrant pas, ne s'inquiét[e] pas outre mesure et ne sui[t] aucun traitement $\aleph^{56}$. Nombre d'articles évoquent aussi l'effroi de patients qui refusent de se faire opérer, quittent l'hôpital et reviennent plus tard dans un état de santé dégradé. Certains patients commencent le traitement mais peuvent l'abandonner par " dégoût » et/ou "indocilité " ${ }^{57}$. Les remarques répétées sur les réactions et le comportement des malades invitent à assimiler l'inculture dont ils témoignent à de la bêtise : certains extraits exceptionnellement développés montrent comment l'objectivité affectée du style peut être détournée. Ce premier exemple 
concerne un curé de soixante-deux ans, venu à Paris pour traiter une « vaste ulcération occupant la partie supérieure de la région dorsale droite » :

Le malade, pour se débarrasser d'une plaie peu douloureuse il est vrai, mais qui gagnait en étendue de jour en jour, consulta un peu tout le monde, des sœurs, des religieuses, des curés de sa connaissance, qui lui appliquèrent des pommades, des onguents et des drogues de toute espèce. [...] Après avoir trainé pendant quelques mois sans consulter de médecin, le malade vint voir M. Andral, qui lui ordonna un traitement tonique, de la liqueur arsenicale de Fowler, et lui conseilla d'aller à Paris et d'entrer à la Maison de santé..$^{58}$

13 Ce curé sortira en moins bonne forme encore de l'hôpital, ce qui n'empêche pas le rédacteur de souligner discrètement via certaines formules (" un peu tout le monde », " après avoir traîné ») que le malade est peut-être responsable de l'évolution alarmante de sa condition. Le second extrait est plus évident. Il s'agit d'un homme de cinquantehuit ans qui voit apparaître « un petit bouton rosé sur le gland de la verge, près du frein prénuptial». Après avoir consulté divers services hospitaliers, l'homme se voit proposer "une opération qui ne fut pas acceptée ». Le style laconique du rédacteur valorise l'effet désastreux de ce refus :

Huit jours après son admission, il reçut son exeat et alla se confier à l'homœopathie. Le gland tout entier se détacha au bout de cinq jours, résultat dont le malade ne manqua pas d'être très-satisfait et qu'il attribuait à la vertu de son nouveau traitement, tandis que ce n'était, à vrai dire, qu'un phénomène consécutif à la marche envahissante du mal..$^{59}$

Le « à vrai dire » du rédacteur met en avant une objectivité qui s'avère ici totalement défectueuse. Incapable de se connaître lui-même, le malade est présenté comme un ignorant de la pire espèce puisqu'il est heureux de constater la désagrégation de sa virilité ; par ailleurs, en rapprochant cet incident de l'évocation de l'homéopathie, le rédacteur n'a pas besoin de commenter l'efficacité de traitements qu'il juge probablement charlatanesques. Ces sous-entendus sont d'autant plus probables que les remarques sur l'intelligence des malades sont courantes: l'intelligence de tel enfant paraît « assez bonne, quoiqu'il ait l'esprit un peu lourd $»^{60}$; rarement, mais cela arrive, le médecin peut se trouver devant "un homme intelligent, rendant parfaitement compte des premiers accidents de sa maladie. $\aleph^{61}$ Plus souvent, les rédacteurs pondèrent ce qu'ils entendent lors de l'anamnèse par des formules telles que « d'après son dire " ou "il/elle s'imaginait». Le patient type, qui peut être un homme, une femme ou un enfant - ces trois catégories sont bien représentées dans la revue - est donc généralement présenté comme peu crédible et potentiellement résistant, ses capacités intellectuelles ne souffrant pas la comparaison avec celles du médecin.

Si les sentiments, émotions et capacités intellectuelles des patients sont occasionnellement notés, les rédacteurs évoquent rarement ce qu'ils éprouvent euxmêmes ${ }^{62}$ et ne formulent pas de jugements moraux : dès lors, ce ne sont pas tant les images ou la technicité de l'écriture qui différencient le plus cette revue de la presse de vulgarisation médicale grand public, que l'absence apparente de positionnement idéologique. Jacques Poirier a bien montré que « les auteurs de vulgarisation médicale ont à opter entre deux registres opposés : rassurer ou terroriser, séduire en rassurant ou au contraire par la terreur, la peur, l'épouvante, l'angoisse, l'inquiétude, la crainte, la menace ("le salutaire effroi") $»^{63}$. Rien de tel ici : le public n'étant pas le même, la revue supprime les considérations morales. Cette posture ne va absolument pas de soi : dans l'étude qu'il consacre à l'hôpital de Rochefort dans la première moitié du XIX ${ }^{\text {ème }}$ siècle, Gregory Beriet signale que «la qualité des maladies appelle des modalités de 
traitement assez différentes ", les blessés bénéficiant de " certaines faveurs » alors que les «galeux et vénériens subissent également certaines défaveurs liées à l'immoralité de leurs maladies " ${ }^{64}$. L'image des hôpitaux que renvoie la Revue photographique n'est pas celle-là: typiquement, les rédacteurs ne formulent pas de jugement sur le comportement sexuel des malades; ils se contentent de noter qu'une affection est survenue chez telle femme "à la suite d'abus de coït " ${ }^{65}$, ou chez tel homme "à la suite d'un coït violent avec une femme aux sécrétions de laquelle il n'était point habitué ${ }^{66}$. Il ne faut toutefois pas s'y tromper: cette neutralité relève d'une démarche idéologique. Si cela était possible, il faudrait comparer ce qu'écrivent les rédacteurs de la revue photographique et ce que fait le personnel hospitalier en présence des malades car, dans la revue, seule la voix du médecin ou de l'interne se fait entendre. On sait pourtant qu'en 1870 les religieuses sont toujours très présentes au chevet des malades et que les infirmières laïques ne bénéficient pas de formation sérieuse : Bourneville, qui codirige la Revue photographique de 1870 à 1873, passera sa carrière à militer en faveur de la structuration et de la laïcisation du métier d'infirmière, qu'il conçoit idéalement pour « des filles du peuple, méritantes et dévouées à la République ${ }^{67}$ afin de mettre fin à «l'insubordination constante des religieuses vis-à-vis des médecins et de l'administration $»{ }^{68}$ Ces éléments sont tus dans la revue, qui simplifie la structure hospitalière et offre un accès direct aux considérations du médecin et au corps du malade car, dans ces pages, la religion n'existe pas et l'histoire de la maladie prime sur l'histoire des individus.

\section{Histoires de maladies}

Médiatiser l'anamnèse a cet effet saisissant de peupler la revue de corps souffrants dont on connaît assez souvent l'identité complète et, dans une moindre mesure, le parcours individuel. L'histoire personnelle de ces malades ne représente cependant qu'une petite portion de la masse des textes, qui s'attachent à détailler ce que les rédacteurs nomment volontiers "l'histoire de la maladie " ${ }^{69}$. N'ayant pas les compétences pour étudier en détail le contenu savant de ces textes, nous nous bornerons à de brèves observations permettant de situer le regard - médiatique - que pose le médecin non plus sur le malade, mais sur la maladie.

17 En premier lieu, placer l'histoire du patient au second plan pour valoriser l'histoire de la maladie revient à inverser l'ordre des priorités naturelles. La "dialectique du paisible et du torturé $»^{70}$ à l'œuvre dans la vulgarisation médicale, qui vise à garantir le bonheur des lecteurs en les éloignant des conduites à risque, est ici délaissée : la Revue photographique présente des cas intéressants indépendamment de ce qu'éprouve le malade. Plutôt que de gloser sur l'indifférence perçue du médecin ${ }^{71}$, insistons sur la conception particulière de la temporalité (médicale et médiatique) qui découle de cette approche : assez brève - généralement un ou deux paragraphes, l'anamnèse couvre le temps long, remontant parfois jusqu'à la naissance du patient et évoquant des incidents ayant pu avoir lieu des années auparavant; passé ce résumé, le texte se dilate pour rendre compte du présent de la maladie. C'est un temps bien plus bref (quelques heures, plusieurs jours) mais qui dans la revue prend la place la plus importante : le texte détaille avec soin et de façon linéaire les observations et interventions. Il n'est pas rare que les événements soient minutés dans un style télégraphique. Or, parce que «le patient n'est par rapport à ce dont il souffre qu'un fait extérieur $»^{72}$, et parce que la 
revue se spécialise dans les cas graves, il est fréquent que l'histoire de la maladie s'achève après l'histoire du malade, l'autopsie tenant lieu de chapitre final plus ou moins détaillé. Le docteur Habran rend compte en 1869 d'un cas d'« endocardite puerpérale typhoïde » : passé l'anamnèse, le texte rend compte au jour le jour de la dégradation de l'état de la malade : fièvre, délire, phlyctènes (cloques), etc., jusqu'à cette dernière entrée datée du 16 février au soir : la malade «a déliré toute la journée ; cependant, elle n'est pas agitée ; elle parait entendre quand on parle, mais ne répond pas. Elle meurt dans la nuit $\aleph^{73}$. Les notations chronologiques s'arrêtent. L'autopsie débute, exclusivement focalisée cette fois-ci sur l'espace physiologique: péricarde, aorte, poumons, foie, rate, reins gauche et droit, intestins, utérus et artères font chacun l'objet d'un paragraphe. L'historique de la maladie cède le pas à l'inventaire de ses effets terminaux. C'est un schéma rédactionnel classique. Notons que le support médiatique dans lequel s'inscrivent ces « histoires » ne laisse pas place au suspense. Les brefs sommaires occasionnellement placés en tête de texte disent tout. Bourneville en est passé maître : « Anévrisme de l'aorte abdominale ; - rupture ; - mort. $»^{74}$; ou encore «Observation. - Grossesse à terme. - Douleurs. - Tentatives obstétricales ; insuccès. -

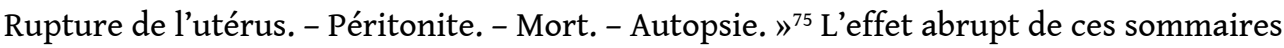
s'augmente parfois de l'usage, typique au sein des périodiques, du fatidique "(à suivre) », qui reporte cruellement la mort annoncée du malade : au même titre que les corps, le texte peut être découpé pour servir les besoins de la revue. L'histoire de la maladie devient alors feuilleton.

En second lieu, l'écriture médicale est difficile à analyser de manière pertinente pour le non spécialiste. Si poétique il y a, elle n'est pas revendiquée, aussi nous garderons-nous de transformer chaque texte de la revue en "Vénus anadyomène ». Cela n'empêche pas l'usage appuyé de l'analogie, vouée à faciliter la compréhension des cas étudiés. Risquons-nous seulement à dire que trois approches paraissent cohabiter sous la plume des rédacteurs de la revue : tout d'abord, on est en présence d'un réseau très fourni d'analogies figées, c'est-à-dire faisant partie du langage courant des spécialistes. On sait par exemple que le dermatologue Jean-Louis Alibert (1768-1837) a volontairement " simplifi[é] le langage de la dermatologie en comparant les maladies avec des objets, animaux et végétaux facilitant [...] la mémorisation des descriptions $\gg^{76}$; cette banalisation de l'analogie à des fins scientifiques cohabite avec une terminologie technique provoquant une "coupure linguistique $»^{77}$ entre les savants et le grand public; enfin, et cela se remarque tout particulièrement dans les pages de la Revue photographique, le rédacteur est parfois en présence de cas si extraordinaires que, faute de termes appropriés, il se voit contraint de créer de nouvelles analogies, ouvrant la voie à des descriptions subjectives. Cela est fréquent en tératologie : pour décrire le cas d'un mort-né anencéphale ( «le cou fait défaut»), le rédacteur évoque la " peau de la face» qui, «en passant sur la poitrine, forme un bourrelet simulant une sorte de collier $»^{78}$. Les maladies ayant atteint une forme extrême favorisent la même approche. Le $«$ je n'ai jamais rien vu de semblable ${ }^{79}$ que certains rédacteurs peuvent occasionnellement formuler les oblige à innover dans l'écriture. L'affection sexuelle de ce patient adepte de l'homéopathie - que nous avons déjà croisé plus haut - contraint le rédacteur à trouver de nouvelles images dans le monde du potager et de la bassecour : «bourrelet en forme de chou-fleur » et induration ayant le « volume d'un œuf de dinde » font système avec l'évocation technique du « champignon qui embrasse toute la verge ", l'urine s'écoulant "librement», "par un grand nombre de points à la fois, comme à travers un arrosoir $»^{80}$. Si la médiatisation de l'anamnèse faisait entrer un peu 
de la vie des malades dans la revue, le regard appuyé que pose le médecin sur l'opacité des corps donne définitivement la priorité aux faits biologiques, que les photographies rendent écrasants.

\section{Du cas d'étude au spectacle pour initiés} que " pour nous, êtres sensibles (et qui ne sommes pas du "métier"), c'est un véritable catalogue de l'horreur $\aleph^{81}$. On reste effectivement hanté par ces images : ce que l'on voit le plus souvent, ce sont les cas tératologiques les plus poussés et des "horreurs " pathologiques inopérables, affligeant des patients ayant perdu forme humaine, dont la vie touche à sa fin (nombre d'entre eux sont morts lorsque paraît le texte), ou qui demeurent infirmes. Cette approche iconographique est déjà celle de la Clinique photographique: le texte s'accompagne d'images représentant des cas dermatologiques extrêmes, comme si la photographie, pour justifier son existence, devait valoir le «coup d'œil ${ }^{82}$. Cette ligne éditoriale perdure pour l'iconographie de la revue, la découverte de l'« insolite » ${ }^{83}$, du «fait très exceptionnel $»^{84}$ ou de la lésion «trèscurieuse $\aleph^{85}$ y étant perpétuellement valorisée. L'association de la modernité de la photographie à la rareté pathologique présente ainsi les hôpitaux de Paris comme des lieux d'avant-garde médicale, la revue devenant pourvoyeuse d'images d'«affection[s] rare[s] dont la nature est encore peu connue ${ }^{86}$.

Il est cependant frappant de constater que les textes s'accommodent mal de ces images. En premier lieu, les photographies sont imprimées sur des planches hors texte, en grand format, seulement accompagnées d'une légende: très faciles à localiser, elles s'extraient d'elles-mêmes de la revue pour captiver le regard. En second lieu, les rédacteurs peinent à les intégrer dans leur propos: très souvent, la planche n'est évoquée qu'en attaque ou à la toute fin de l'article : le corps du texte se lit très bien sans les images car les descriptions abondent. Si son exactitude est constamment revendiquée, la photographie ne sert donc guère qu'à " donner une idée " ${ }^{87}$ ou valider par un « coup d'œil » le propos du rédacteur.

21 L'image elle-même phagocyte parfois le texte : ainsi de ces deux cas d'encéphaloïde de la région parotidienne (tumeur située au niveau des glandes salivaires), dont «l'histoire [...] n'offr[e] rien de particulier $»^{88}$ mais dont le gigantisme justifie la présence de quatre planches photographiques pour sept lignes de texte. Ailleurs, on trouve une planche qui « représente un malade qui a succombé à une asphyxie lente déterminée par des adénomes multiples. » Le texte de trois lignes s'achève sur cette seule remarque : " ce cas pathologique offre un grand intérêt par sa rareté. $»^{89}$ Mais de quelle rareté parle-t-on? Si "l'histoire " était intéressante médicalement parlant, ne serait-elle pas racontée ? Tout porte à croire que l'on parle de rareté visuelle. Or, livrée sans texte ou presque, donnée sans motivation médicale précise, cette image appelle d'elle-même des considérations d'ordre esthétique. Il apparaît à de rares occasions que le rédacteur souligne cette valeur dans l'image qu'il commente. C'est flagrant ici :

La planche VII représente une jeune fille de seize ans, atteinte de carie de la première côte avec ouverture spontanée de l'abcès dans la région sternoclaviculaire droite. Cette fille peut être considérée comme offrant le type parfait de la constitution dite scrofuleuse. La physionomie douce et mélancolique s'accuse dans le regard et l'expression de la bouche; si l'on ajoute à cela l'existence d'un abcès froid, les lésions remarquables des mains (planches VIII et IX), chez lesquelles 
la strume a détruit ou déformé plusieurs phalanges, l'engorgement des ganglions du cou, de l'aisselle ou de l'aine, une leucorrhée abondante, on a réuni tous les caractères du lymphatisme à sa plus haute expression. ${ }^{90}$

Trois planches pour onze lignes d'un texte qui brouille totalement la frontière entre l'étude du «beau cas» (formule fréquente), c'est-à-dire du cas «type » indiqué par la légende, et la critique artistique : succédant à une première phrase écrite dans un style tout à fait attendu, le passage détaillant les traits de la jeune fille introduit le doute sur les motivations du rédacteur, qui se comporte comme un visiteur au musée: l'expression "on a réuni» transforme la jeune fille en sujet de tableau (quel est l'auteur? Dieu? Le médecin? Le photographe ?), tandis que le « lymphatisme à sa plus haute expression" sonne comme un mouvement concurrent de l'impressionnisme. L'écriture soulignant de manière appuyée la féminité de la malade, il est probable que le rédacteur y voit une image partiellement érotique (épaules dénudées, bandeau dans les cheveux, « douceur » de la physionomie).

Fig. 1

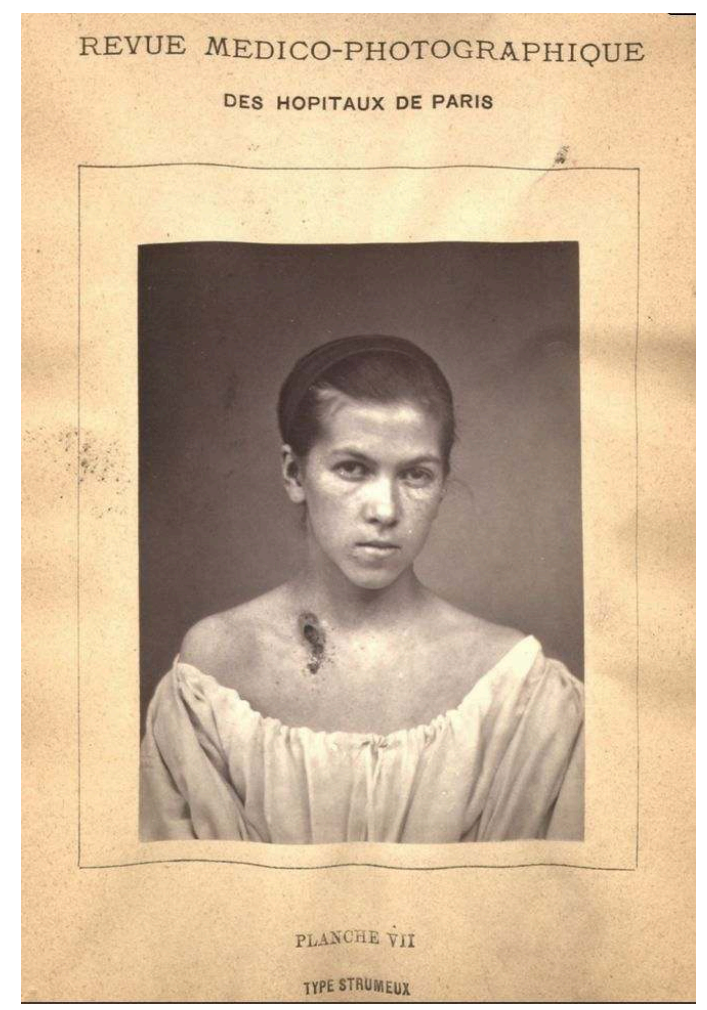

Revue médico-photographique des hôpitaux de Paris, « planche VII. Type strumeux », 1876 (située entre les p. 40 et 41 du volume)

Les cas tératologiques relèvent totalement de cette logique de l'image-spectacle ou de l'image-tableau, mais leur traitement est désordonné. Certains cas sont discrets (les patients peuvent vivre avec), d'autres sont extrêmes (la personne est infirme ou le bébé est mort-né), ils peuvent être accompagnés de textes longs ou très brefs, être parisiens, provinciaux ou étrangers : pour les cas tératologiques les plus graves, la revue fait volontiers appel aux collègues de l'étranger pour proposer des planches - plusieurs la plupart du temps. Court ou long, le texte use toujours d'un registre médical traversé de connotations esthétiques: le visage de ce mort-né anencéphale «rappelle immédiatement la physionomie hideuse du crapaud $»^{91}$, tandis que le petit Adolphe X..., 
enfant de deux ans dont les bras et une jambe ne sont pas développés, "présente une petite figure charmante $\aleph^{92}$. Sur la photographie, on le voit esquisser un début de sourire (le seul de la revue). La tératologie autorise l'investissement émotionnel du rédacteur, qui manque souvent de lucidité dans la sélection des informations : signé "La Rédaction", un texte de presque deux pages paru en 1869 évoque le cas d'un Portugais «ayant successivement parcouru diverses contrées de l'Europe " pour être observé par les médecins. Cet homme possède trois jambes et deux appareils génitaux masculins parfaitement développés: si la conformation de la troisième jambe est détaillée, le rédacteur ne dit pas comment il se déplace, ni comment il va "à la garderobe "; en revanche, près de la moitié du texte est consacrée aux parties sexuelles : le fait qu'elles soient pleinement fonctionnelles et «d'un calibre imposant " pousse le rédacteur à détailler la façon dont cet homme emploie "la richesse de ses moyens » lors du coït. Parler de richesse pour un tel cas, c'est privilégier la part de fantasme dans l'observation, chose encouragée par l'image, centrée sur les deux verges, les effets de flou ne laissant aucun doute sur le fait que ce deuxième pénis paraît nettement plus porteur qu'une troisième jambe. Impossible de s'y tromper : il ne s'agit pas d'un patient mais d'un cas " assez rare, et c'est surtout à ce titre que s'y rattache un certain intérêt de curiosité ${ }^{93}$. Le rédacteur ne se comporte plus en médecin mais en amateur. On sait que les collections de cires, gravures et photographies sont fréquentes à cette période ${ }^{94}$, que ce soit dans les musées médicaux ou les collections personnelles de médecins (Alfred Hardy possède par exemple une belle "collection de nez $»^{95}$ au musée de l'hôpital Saint-Louis); on sait qu'elles induisent une certaine sociabilité chez les spécialistes, qui ne manque pas d'être signalée dans les divers périodiques spécialisés. Dans la Revue photographique, les médecins se montrent les malades qu'ils ont découverts et les cas tératologiques s'inscrivent dans des séries: le texte, signé « $\mathrm{La}$ Rédaction ", conclut en précisant que cette image relève d'une "collection » et qu'un « fait analogue » sera « donné » accompagné d'une « note ${ }^{96}$ dans le prochain numéro. Il s'agit en l'occurrence d'une petite fille de dix ans, qui a trois jambes aussi: photographiée nue, de face puis de dos, elle se maintient difficilement sur les accoudoirs d'un fauteuil. Elle porte des guêtres aux trois membres - certains sont équipés d'un dispositif de redressement ou de maintien. Elle regarde directement l'objectif sans sourire. Sur sa tête, une couronne de fleurs ${ }^{97}$. L'image mélange deux représentations : cette petite fille est une princesse, ce cas médical est un «monstre ». L'impact est maximal.

Que faire de ces images ? La tentation de l'interprétation est lancinante : le mobilier, la nudité totale ou partielle, les draps et tenues d'hôpital, les vêtements de ville, les accessoires (colliers, bijoux, coiffes), la posture, l'âge, le sexe, le regard frontal ou détourné, le cadrage serré ou non, les effets de flou, tous ces éléments invitent à l'interprétation esthétique. Telle posture d'un cas rachitique évoque perversement $L a$ Naissance de Vénus de Botticelli ${ }^{98}$, tandis que la nudité répétitive des corps confère à cette iconographie des connotations érotiques allant de la peinture classique à la pornographie. Frédérique Calcagno-Tristant propose une telle lecture pour quelquesuns des clichés réalisés par Montméja pour la Clinique photographique ${ }^{99}$; cette approche pourrait être pareillement adoptée pour la Revue photographique, en dépit de l'anonymat des photographes. Preuve que ces images forcent à réagir/interpréter, l'un des volumes a fait l'objet d'une intervention de lecteur (de quelle époque ?): dans celui de 1874, numérisé par Google, le visage d'une femme affligée d'un mamelon double ${ }^{100}$ a été étrangement raturé. La personne était-elle choquée ? Voulait-elle préserver l'anonymat 
d'une personne trop dénudée ? Pourquoi être intervenu sur ce cas seulement ? De fil en aiguille, d'autres questions se posent: ne s'agirait-il pas plutôt d'un griffonnage érotique? Dans sa forme, le "loup» dessiné est effectivement un cache-sexe au maillage étonnamment travaillé... Le vertige interprétatif est sans fin parce qu'il nous renvoie à nos propres conceptions du normal, du pathologique et de l'érotique.

Fig. 2

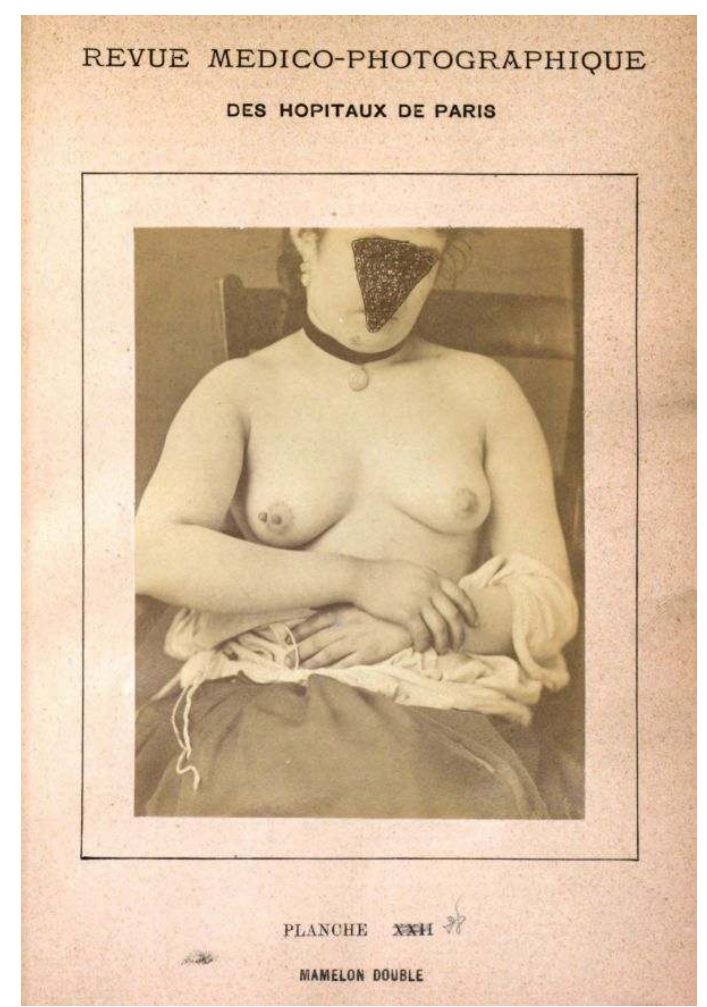

Revue médico-photographique des hôpitaux de Paris, "Planche XXII. Mamelon double », 1874 située entre les p. 180 et 181 
Fig. 3

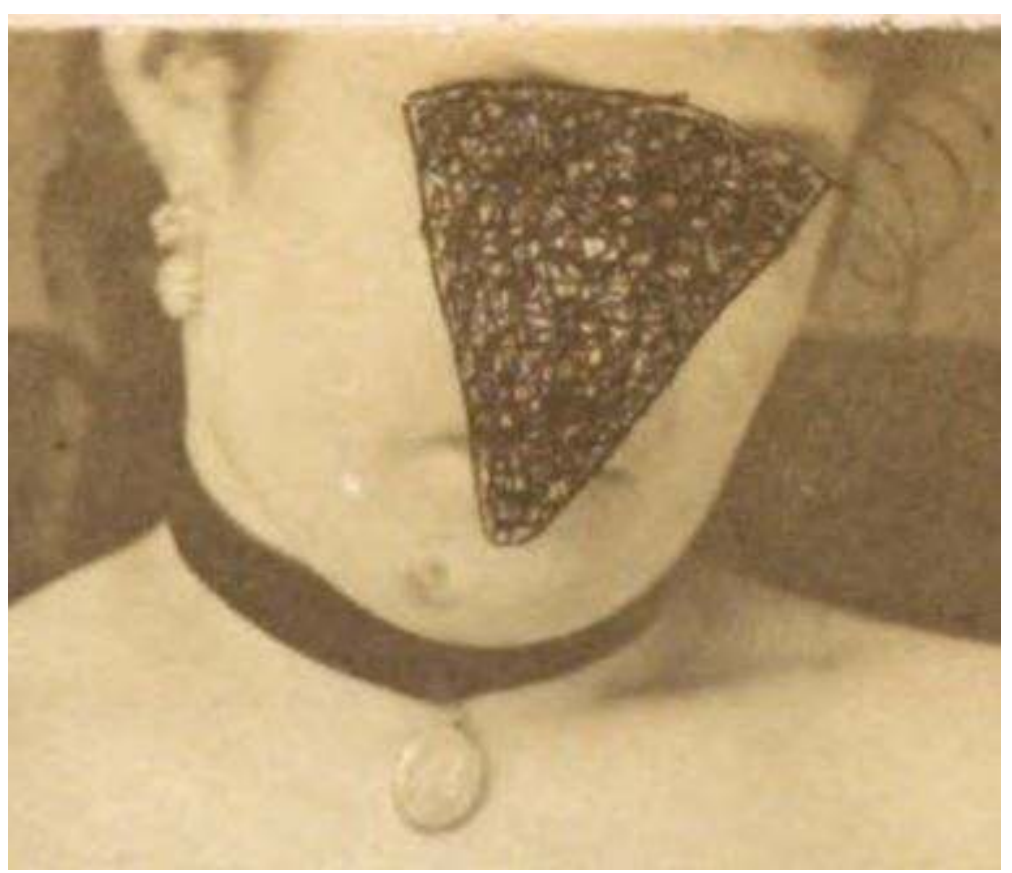

Revue médico-photographique des hôpitaux de Paris, «Planche XXII. Mamelon double », 1874, située entre les p. 180 et 181 (détail)

Selon nous, il y a trop de relais dans la production de ces photographies pour que l'on puisse en donner une interprétation définitive : le rédacteur n'est pas le photographe, le photographe est anonyme dans la revue, et il arrive assez souvent que les photographies aient été réalisées ailleurs qu'à l'hôpital Saint-Louis. Marie Barthélémy précise que, avant l'arrivée de l'«artiste dermatographe" Félix Méheux, les photographies médicales de l'hôpital Saint-Louis «étaient surtout réalisées par du personnel interne $»^{101}$; quant à l'intention d'Arthur de Montméja, qui signe effectivement toutes les photographies de la Clinique photographique mais semble n'en produire aucune pour la revue, il nous paraît très délicat d'affirmer qu'elle encourage " une déontologie professionnelle dont il ressort une attitude morale et médicale à tenir vis-à-vis du patient. ${ }^{102}$ Les avant-propos de Montméja et la ligne éditoriale certes squelettique - de sa revue inviteraient plutôt à considérer sa signature comme une marque de distinction sociale et/ou d'appropriation de la photographie médicale (en son nom surtout et en celui des hôpitaux parisiens). Quant à la démarche artistique, elle est tout simplement impossible à évaluer, même chez Montméja, qui emploie certes des analogies artistiques ( « ma main s'habitua à tenir le pinceau que guidait l'œil du maître $»^{103}$ - en l'occurrence Alfred Hardy), mais elles se bornent à reproduire le lieu commun d'une photographie inféodée aux besoins scientifiques. Sachant qu'à ce moment les seuls modèles de portraits préexistants sont issus des ateliers de photographes traditionnels ou de l'atlas physionomique de Duchenne de Boulogne, on ne saurait dire si les planches produites pour la Clinique et la revue sont le fruit d'une intention d'imitation, d'adaptation, de distanciation ou de jeu sur les pratiques des photographes traditionnels : le patient regarde-t-il l'objectif parce qu'il le veut ou parce qu'on le lui a demandé ? La malade a-t-elle insisté pour conserver ses bijoux ? A-t-elle oublié de les enlever? Le photographe et/ou le médecin lui ont-ils demandé de les garder? Pourquoi? Lorsque le médecin a fait procéder à la prise de vue, voulait-il 
immortaliser un cas curieux ou une patiente ou un patient qui lui a plu - humainement ou physiquement? Était-ce initialement pour une communication publique ou pour une collection personnelle? Et le photographe : était-il compatissant, dégoûté, amusé, fasciné, indifférent? A-t-il voulu traduire cela dans la photographie? Voulait-il valoriser la personne ou la maladie? Aucun témoignage n'existe. C'est ce qui rend ces images d'autant plus magnétiques. Planche après planche, la même question demeure : comment a-t-on pu produire « ça »?

Dès lors, une seule certitude demeure: ces images témoignent de métiers en construction - le métier de photographe et celui de médecin, les images paraissant influencées par des techniques et des regards artistiques, forains et médicaux, mais dans des proportions impossibles à établir. Mises à nu sans protocole stable, révélées dans toute leur intimité biologique, leur visage et leur nom parfois complètement dévoilés, les personnes présentées dans ces planches apparaissent comme les victimes collatérales d'une revendication impérieuse, porteuse de progrès mais assénée avec une brutalité totale : rien, ni la pudeur des individus ni les interdits sociaux ou religieux, ne saurait barrer la route au regard des médecins - et peu importe si certaines de ses connotations sont condamnables.

\section{De Saint-Louis à la Salpêtrière : les errances de la photographie médicale}

On ignore si la Revue photographique des hôpitaux de Paris a connu un succès d'estime et/ ou commercial. On peut cependant dire que, sans être honteuse, sa longévité (1869-1876) est faible comparée à d'autres périodiques spécialisés, qui peuvent cumuler plusieurs décennies d'existence ${ }^{104}$. La ligne éditoriale pose clairement problème dès les débuts: le contenu du tout premier volume, de loin le plus homogène, encourage à penser que Montméja et Rengade souhaitent en premier lieu que les articles se focalisent sur un seul cas, idéalement rare, accompagné de photographies; lorsque Bourneville prend en 1870 la place de Rengade au poste de corédacteur en chef, ce souhait paraît moins prioritaire: s'intéressant visiblement plus aux approches comparatives, il focalise davantage ses articles sur une maladie, ce qui permet d'évoquer simultanément plusieurs patients dont les symptômes ne sont pas forcément d'une grande rareté. Pourtant, les photographies continuent de présenter des cas extrêmes. La ligne éditoriale ne trouve à aucun moment sa résolution, d'autant plus que les rédacteurs de province et de l'étranger (Lyon et Lausanne notamment) sont de plus en plus représentés, ce qui dilue un peu l'identité parisienne du périodique. On peut supposer que le périodique peine à trouver sa voie par manque d'investissement de la part de Montméja : lorsque Rengade quitte la codirection du titre, Montméja est effectivement en plein travail sur le Traité des opérations qui se pratiquent sur l'œil (1871) et n'a probablement pas le temps de s'investir outre mesure dans la Revue photographique - c'est peut-être l'une des raisons pour lesquelles il ne signe presque aucun texte et aucune des photographies qui y sont publiés. Succédant à Rengade, Bourneville a pu profiter de sa position pour occuper les pages du périodique. On ne connaît pas les échanges qu'ont eus les deux rédacteurs en chef, mais on constate qu'en 1873 Bourneville fonde Le Progrès médical et que Montméja reprend seul le contrôle du périodique, qu'il renomme alors Revue médico-photographique des hôpitaux de Paris. Outre 
la revendication d'une reprise en main personnelle, ce changement de titre est un aveu que la photographie peine à assumer le rôle de composante structurante pour la revue.

À l'incohérence partielle de la ligne éditoriale s'ajoute un second facteur. Dans les années 1860, l'hôpital Saint-Louis développe simultanément deux méthodes pour représenter la maladie : la photographie et la céroplastie. Jules Baretta produit son premier moulage exposé en 1867, au moment même où paraît la Clinique photographique ${ }^{105}$. Or, lorsque Montméja et Rengade lancent la Revue photographique en 1869, ils affirment que la " vérité » de la photographie « est toujours supérieure à celle de tout autre genre iconographique $»^{106}$. En contexte, cette affirmation n'est certainement pas triomphante, mais défensive. Alfred Hardy lui-même se désintéresse très vite de la photographie: dans le compte rendu de la séance du 19 janvier 1869 de l'Académie impériale de médecine, L'Abeille médicale, précise certes que l'éminent médecin invite ses confrères à «venir faire photographier les cas anatomopathologiques les plus intéressants qu'ils auront observés dans leurs services ». On y lit surtout ceci :

Mais, ajoute M. Hardy, on a trouvé mieux que la photographie pour la représentation fidèle de ces maladies [de la peau]. Un artiste éminent, M. Baretta, a imaginé un procédé de moulage, à l'aide d'une pâte, dont la composition est restée le secret de l'inventeur, qui reproduit une image des maladies de la peau aussi parfaite que nature. ${ }^{107}$

Voilà un cinglant désaveu, alors même que la Revue photographique commence à peine à paraître. La Revue photographique reprend d'ailleurs ce texte mais expurge toute la partie évoquant Baretta ${ }^{108}$. Comment faire autrement sans perdre la face? Si la photographie donne toute sa personnalité à la revue, elle l'a donc probablement placée d'emblée dans une position délicate, qui se vérifie dans l'hétérogénéité grandissante des images: à compter de 1873, soit par manque de matière, soit par manque d'investissement, la revue reproduit de plus en plus de photographies de moulages de cire, ainsi que des gravures ou des dessins d'Alibert.

30 Le périodique périclite brutalement en 1876. L'incohérence grandissante de la ligne éditoriale et la légitimité certainement mal établie de la pratique photographique à l'hôpital suffiraient à justifier cette disparition. Un troisième facteur, plus hypothétique, doit cependant être évoqué : fort d'une légion d'honneur obtenue pour ses états de services militaires ${ }^{109}$ et suffisamment riche pour entreprendre la restauration d'un château à Carlux - en Dordogne ${ }^{110}$, Arthur de Montméja disparaît brutalement de la vie médiatique en même temps que sa revue. Or, en mars 1876 éclate l'affaire Garrigue, dans laquelle un certain docteur Montméja, "vieillard au chef branlant, à la taille voûtée, aux jambes fléchissantes » ${ }^{111}$, joue un rôle fort peu glorieux : alors que l'on soupçonne la famille d'avoir empoisonné le défunt, on apprend que ce médecin a été appelé au chevet du malade plusieurs jours avant sa mort mais n'a pas pris les dispositions nécessaires à la survie de l'homme. ${ }^{112}$ L'affaire se déroule aussi en Dordogne et l'on sait que le père d'Arthur de Montméja pratique la médecine du côté de Sarlat. ${ }^{113}$ Dès lors, tout semble indiquer qu'il s'agit bien de Montméja père. Très suivie en France et dans les journaux parisiens ${ }^{114}$, l'affaire aurait-elle provoqué le déshonneur et la chute brutale de la famille Montméja?

Il semblerait dès lors que la Revue photographique des hôpitaux de Paris ait été au cœur de l'un des concours de circonstances les plus magistralement désastreux de l'histoire de la presse : lancée au moment précis où la céroplastie triomphe de la photographie, cette 
revue mal dirigée disparaît au moment même où son rédacteur en chef connaît peutêtre le discrédit. Et la série continue : toujours en 1876, Alfred Hardy change de service, passant de l'hôpital Saint-Louis à l'hôpital Necker ${ }^{115}$, tandis que Bourneville, qui remplit « officieusement [...] les fonctions d'assistant $»^{116}$ de Charcot depuis 1871, est nommé à la Salpêtrière: au moment où Montméja est à la peine, son mentor disparaît et Bourneville lance l'Iconographie photographique de la Salpêtrière, qui se détourne des cas anatomo-pathologiques pour réassigner à la photographie la charge de reproduire la physionomie de patients souffrant d'atteintes neurologiques et mentales ${ }^{117}$.

À ce moment, les enjeux de la photographie médicale sont donc redirigés, permettant à la Salpêtrière de saisir la place médiatique préalablement occupée par l'hôpital SaintLouis. Devenu Directeur du service photographique de la Salpêtrière en 1882, le très médiatique Albert Londe veillera d'ailleurs à reléguer Montméja et sa revue aux oubliettes de l'histoire: partisan jusqu'à la mauvaise foi, il écrit sans ciller que "l'entrée officielle de la Photographie dans les services hospitaliers date de l'année $1878 »^{118}$ - moment où Charcot obtient de l'APHP que soit créé un service photographique à la Salpêtrière. Cette affirmation erronée colorera durablement les approches historiques de la photographie médicale. De Montméja à Londe, on constate ici que ce ne sont pas tant les institutions que les hommes travaillant à leur service qui ont voulu s'approprier la photographie médicale. Sans grand succès semble-t-il : d'un pôle parisien à un autre, des corps malades de l'hôpital Saint-Louis aux hystériques de la Salpêtrière, la photographie médicale ne trouvera pas davantage sa voie, à tel point que, malgré le prestige de Charcot, Albert Londe et son collègue Paul Richer finiront par renoncer aux corps malades - Londe parle plutôt de "l'étude du nu pathologique $»^{119}$ - pour renouer avec les corps plus traditionnels du monde artistique $^{120}$.

La Revue photographique des hôpitaux de Paris est un objet trouble. S'attribuant le rôle de vitrine pour les hôpitaux de Paris, elle met en scène la rencontre de deux milieux diamétralement opposés: le public médical, éduqué et privilégié, et les cas pathologiques les plus exceptionnels du Paris populaire. S'inscrivant dans une modernité typique du Second Empire, où la centralisation et la spectacularisation des merveilles de la science et de l'industrie sont la règle, elle remplit plus ou moins consciemment trois fonctions: offrant un panorama saisissant de la vie du Paris populaire et de ses accidents biologiques (1), elle veut raconter des «histoires" de maladies sélectionnées pour leur rareté (2), en les accompagnant d'une iconographie qu'elle érige en argument de vente principal via son titre (3). Faute de ligne éditoriale suffisamment développée, cette revue est d'une extrême ambiguïté : le ton mondain employé dans l'avant-propos oriente la réception de l'ensemble du contenu, si bien que l'intérêt des cas présentés dépasse très largement le seul cadre du partage des savoirs, pour flatter la curiosité de lecteurs faisant partie d'une même communauté d'initiés. Tous ces éléments augmentent l'impact d'images dont l'intérêt médical peine parfois à être démontré dans le texte. On ignore dans quelle proportion la communauté médicale a apprécié - ou non - cette proposition médiatique, mais les dysfonctionnements de la ligne éditoriale et le comportement de ses collaborateurs (Rengade, Bourneville, Hardy dans une moindre mesure) indiquent que Montméja s'est trouvé de plus en plus isolé au sein de sa revue. Au-delà du jour peu flatteur que cette enquête paraît jeter sur Arthur de Montméja, et malgré une représentativité très difficile à évaluer, cette revue est un témoignage important du regard que peut porter la médecine sur des corps impossibles à connaître autrement qu'en se confrontant à leur opacité. Quelques années plus tard, 
les rayons $\mathrm{X}$ marqueront l'avènement de l'homme transparent, ouvrant la voie à de nouvelles perceptions médicales et plus largement culturelles du corps et de la réalité qui nous entoure.

\section{NOTES}

1. Arthur de Montméja et Alfred Hardy, Clinique photographique de l'hôpital Saint-Louis, Paris, Librairie Chamerot et Lauwereyns, 1868, p. II (préface).

2. Ibid.

3. Voir Michel Frizot, Nouvelle Histoire de la photographie, Paris, Larousse, 2001, p. 262 ; Denis Canguilhem, Le Merveilleux scientifique, Paris, Gallimard, 2004, p. 32-35 ; Magali Le Mens et JeanLuc Nancy, L'Hermaphrodite de Nadar, Saint-Étienne, Créaphis, 2009 ; Catherine Mathon (dir.), Duchenne de Boulogne, Paris, École nationale supérieure des beaux-arts, 1999 ; Monique Sicard, La Fabrique du regard, Paris, Odile Jacob, 1998, p. 115-126 ; Monique Sicard, « Duchenne de Boulogne, médecin-photographe (1806-1875)», Institut des textes et manuscrits modernes, en ligne: http://www.item.ens.fr/articles-en-ligne/duchenne-de-boulogne-medecin-

photographe-1806-1875/.

4. Arthur de Montméja et Jules Rengade, «Avant-propos », Revue photographique des hôpitaux de Paris, $1869, \mathrm{np}$.

5. Les deux auteurs emploient le terme en couverture (Duchenne de Boulogne) et dans l'avantpropos (Hardy et Montméja).

6. Ces 14 livraisons paraissent à compter du printemps 1867. Réunissant 49 photographies à l'albumine rehaussées de couleurs, cet ensemble a ensuite été réédité à trois reprises sous forme d'ouvrage de librairie augmenté de 11 photographies supplémentaires. Voir Denis Canguilhem, op. cit., p. 171-172.

7. Arthur de Montméja et Alfred Hardy, op. cit. p. II.

8. Ibid., p. I.

9. Ibid., p. II-III.

10. Ibid., p. III

11. Ibid., p. I.

12. Arthur de Montméja et Jules Rengade, op. cit.

13. Ibid.

14. Quelques exemples de titres paraissant en 1869 : La France médicale et pharmaceutique, Bulletin général de thérapeutique, Archives générales de médecine, Gazette hebdomadaire de médecine et de chirurgie, Gazette médicale, Journal des connaissances médicales pratiques et de pharmacologie, Revue de thérapeutique médico-chirurgicale, Journal de chimie médicale, Répertoire de pharmacie, Revue médicale française et étrangère, revue thérapeutique du Midi, etc.

15. Si le Moniteur des hôpitaux: revue médico-pharmaceutique de Paris cesse de paraître en 1859, le Bulletin et mémoires de la Société médicale des hôpitaux de Paris et la Gazette des hôpitaux civils et militaires occupent parfaitement le terrain, Armand Husson leur adjoignant en 1867 le bulletin de Statistique médicale des hôpitaux de Paris.

16. La Société de biologie, l'Académie nationale de médecine, la Société anatomique de Paris, la Société clinique de Paris, la Société de chirurgie de Paris, sans parler de nombreuses sociétés savantes de province, publient leurs propres comptes rendus de séance. 
17. Arthur de Montméja et Jules Rengade, op. cit., np.

18. Le Musée des sciences propose par exemple un frontispice à la gloire des « maîtres de la science moderne » (voir le premier volume, paru en 1855).

19. Rengade a publié quelques mois auparavant son roman des Aventures extraordinaires de Trinitus (Paris, Hetzel, 1873).

20. Ibid.

21. Nous paraphrasons le titre de l'ouvrage d'Elina Absalyamova et Valérie Stiénon (dir.), Les voix du lecteur dans la presse française au XIXe siècle, Limoge, PULIM, 2018. Globalement, les publications visant le public médical sont volontiers hebdomadaires et revendiquent leur statut de journal ou de gazette. Elles sont en contact direct avec l'actualité (pratique de la médecine en ville, à la campagne, nouveautés thérapeutiques, concours de recrutement, etc.) : la plupart bruissent de voix, celles du lecteur, des contributeurs occasionnels et souvent du rédacteur en chef (c'est le cas de L'Abeille médicale) ; un autre modèle serait celui de la Gazette hebdomadaire de médecine et de chirurgie (publiée chez Victor Masson), qui en 1869 se construit selon l'exemple de la toute nouvelle Revue des cours scientifiques de la France et de l'étranger (publiée chez Germer Baillière) : la maquette est la même et elle consigne divers cours, conférences et études originales, tout en offrant une place de choix à la correspondance. La Revue photographique des hôpitaux de Paris est publiée quant à elle chez Adrien Delahaye, libraire-éditeur situé place de l'École de Médecine : notre sujet n'est pas de parler des stratégies de distinction chez les éditeurs de périodiques, mais le terrain est visiblement fécond, comme l'a déjà montré Viéra Rebolledo-Dhuin pour le livre («Le paysage éditorial de médecine à Paris», Histoire des sciences médicales, t. LI, n², 2017, p. 175-190).

22. Un bulletin de société savante vaut généralement de 50 à 75 centimes ; par ailleurs, la revue est mensuelle, de format in- $8^{\circ}$, ses livraisons comptent initialement 16 pages accompagnées de trois planches photographiques. L'abonnement parisien coûte 20 francs. Les volumes ne comportent pas ces informations: il faut consulter les réclames parues dans les numéros de L'Abeille médicale de 1869 pour les trouver.

23. Arthur de Montméja et Jules Rengade, « Avant-Propos », op. cit., 1869, np.

24. Nous raccourcissons parfois les titres pour alléger: Revue photographique pour Revue photographique des hôpitaux de Paris, Clinique photographique pour Clinique photographique de l'hôpital Saint-Louis.

25. Arthur de Montméja et Jules Rengade, « Avant-Propos », op. cit.

26. Le monde hospitalier lui-même tire pleinement parti de la politique menée par Napoléon III : lorsque paraît la Revue photographique, la reconstruction de l'Hôtel-Dieu de Paris est entamée depuis un peu plus de deux ans.

27. Jules Mesnard, Les Merveilles de l'Exposition universelle de 1867, Paris, Lahure, 1867, p. 5.

28. Olivier Faure, «Le regard des médecins", dans Alain Corbin (dir.), Histoire du corps, Paris, Seuil, « Points », 2005, vol. 2, p. 22.

29. Michel Foucault, Naissance de la clinique, Paris, PUF, 2007 [1963], p. 85.

30. Foucault donne deux exemples de pratique, la première rendant fidèlement compte du plan suivi dans la plupart des textes de la revue : «À la clinique d'Edimbourg, l'observation clinique consistait en quatre séries de questions: la première sur l'âge, le sexe, le tempérament, la profession du malade; la seconde sur les symptômes qu'il éprouvait; la troisième concernait l'origine et le développement de la maladie ; la quatrième enfin portait sur les causes éloignées et les accidents antérieurs. » (ibid., p. 111)

31. Revue photographique des hôpitaux de Paris, 1870, p. 29.

32. «Pathologie externe », ibid., p. 49.

33. Bourneville, « Note sur le tænia, ses effets, son traitement », ibid., p. 64.

34. Bourneville, «Syphiolographie », ibid., p. 104.

35. «Pathologie », ibid., p. 194. 
36. «Thérapeutique », ibid., p. 211.

37. «Clinique obstétricale », ibid., 1871, p. 25.

38. E. Dupouy, « Pathologie externe », ibid., p. 36.

39. Ibid., 1875, p. 1.

40. Dr. Abadie, ibid., 1875, p. 37.

41. « Traitement de la chute complète de l'utérus », ibid., p. 41.

42. « Mycosis ", ibid., p. 57.

43. Ibid., p. 271.

44. Arthur de Montméja, « Tumeur des sacs lacrymaux », ibid., 1871, p. 265.

45. Ibid., p. 52.

46. Ibid., 1875, p. 46.

47. «Observation de lichen hypertrophique », ibid., 1869, p. 39.

48. J. Lucas-Championnière, "Trépanation du crâne par une fracture de la voûte sans plaie communicante ; guérison ", ibid., 1875, p. 21.

49. Ibid., p. 20

50. E. Dupuy, Revue photographique des hôpitaux de Paris, 1871, p. 36.

51. «Traitement du rhumatisme cérébral par les bains froids ", ibid., 1875, p. 28.

52. Michel Foucault, op. cit., p. 30-31.

53. La revue ne se soucie pas de clarifier le fonctionnement hospitalier, déjà connu de ses lecteurs, et ne cherche pas la représentativité des cas. Claire Barillé donne un panorama autrement plus complet de ce fonctionnement, ainsi que des statistiques précises : Claire Barillé, «L'hôpital à Paris dans les quartiers populaires », dans Elisabeth Belmas et Serenella NonnisVigilante (dir.), La Santé des populations civiles et militaires. Nouvelles approches et nouvelles sources hospitalières, XVII ${ }^{e}$-XVIII ${ }^{e}$ siècles, Villeneuve d'Ascq, Presses universitaires du Septentrion, 2010, p. 209-218.

54. «Eléphantiasis de la verge et du scrotum », Revue photographique des hôpitaux de Paris, 1869, p. 1.

55. "Lipômes symétriques ", ibid., p. 54.

56. Ibid., p. 59.

57. Voir ce cas qui cumule les deux : ibid., 1875, p. 47.

58. " Mycosis ", Revue photographique des hôpitaux de Paris, 1875, p. 57.

59. « Epithélioma de la verge », ibid., 1870, p. 25-26

60. Dr. Waller, « Observation de paralysie musculaire pseudo-hypertophique », ibid., 1875, p. 150.

61. E. Vidal, «Lèpre tuberculeuse tachetée et anesthésique », Ibid., 1875, p. 120.

62. Quelques occurrences existent: un médecin évoque par exemple l'odeur «horrible, nauséeuse, suffocante » que dégage une malade (Alfred Fournier, «Végétations monstrueuses de la vulve ", ibid., 1869, p. 18); un autre conclut son texte sur la question du dégoût : « [...] y a-t-il pour une jeune femme une infirmité plus dégoûtante que celle d'une fistule recto vaginale, et ne faut-il point à tout prix l'en débarrasser ? » (Demarquay, ibid., 1875, p. 3).

63. Jacques Poirier, «La santé en images à la fin du XIx ${ }^{\mathrm{e}}$ siècle. L'iconographie de la littérature de vulgarisation médicale de 1870 à 1914 ", dans Bernadette Bensaude-Vincent et Anne Rasmussen (dir.), La Science populaire dans la presse et l'édition, Paris, CNRS Éditions, 1997, p. 103-119. L'auteur signale par ailleurs que Jules Rengade, premier codirecteur de la revue photographique, aime à terroriser son lectorat dans ses écrits de vulgarisation.

64. Grégory Beriet, «Le corps malade et souffrant: vivre, survivre et mourir à l'hôpital (Rochefort, fin XVIII ${ }^{\mathrm{e}}$ siècle-milieu du XIX ${ }^{\mathrm{e}}$ siècle) », dans Frédéric Chauvaud (dir.), Corps saccagés, Rennes, PUR, 2009, p. 139-149, en ligne : https://books.openedition.org/pur/98925.

65. Revue photographique des hôpitaux de Paris, 1875, p. 180

66. Arthur de Montméja, «Eczéma des parties génitales », Ibid., 1870, p. 85. 
67. Véronique Leroux Hugon, Jacques Poirier et Philippe Ricou, «L'histoire de l'École d'infirmières de la Salpêtrière ", Histoire des sciences médicales, 1997, t. XXXI, n², p. 189-200, ici p. 191.

68. Désiré-Magloire Bourneville, Laïcisation de l'assistance publique. Conférence faite à l'Association philotechnique le 26 décembre 1880, Paris, au Bureaux du Progrès médical, 1881, p. 18.

69. Un exemple typique Revue photographique des hôpitaux de Paris, 1875, p. 131.

70. Jacques Poirier, op. cit.

71. Voir Olivier Faure, op. cit., p. 26.

72. Michel Foucault, op. cit., p. 6.

73. Dr. Habran, «Endocardite puerpérale typhoïde », Revue photographique des hôpitaux de Paris, 1869, p. 183

74. Bourneville, ibid., 1869, p. 100.

75. Bourneville, « Notes et observations sur quelques maladies puerpérales », ibid., 1871, p. 25.

76. Gérard Tilles et Estelle Lambert, «L'image pédagogique, innovation de la dermatologie au XIX siècle ", Le Musée de l'hôpital Saint-Louis. Une exposition virtuelle de la BIU Santé, mise en ligne le 18 octobre 2017, [en ligne], URL : https://www.biusante.parisdescartes.fr/stlouis/fr/02-03.htm ; voir aussi Frédérique Calcagno-Tristant, «Dermatologie du sensible », Protée, automne 2004, vol. 32, n², p. 85-97.

77. Yves Jeanneret, Écrire la science. Formes et enjeux de la vulgarisation, Paris, PUF, 1994, p. 98.

78. «Anencéphalie ", Revue photographique des hôpitaux de Paris, 1870, p. 32.

79. Par exemple: "Vices de conformation nombreux et graves observés chez un nouveau-né », ibid., 1869, p. 93.

80. Ibid., 1870, p. 27.

81. Georges Didi-Huberman, Invention de l'hystérie. Charcot et l'Iconographie photographique de la Salpêtrière, Paris, Macula, 1982, p. 37.

82. Revue photographique des hôpitaux de Paris, 1869, p. 179.

83. Ibid., 1870, p. 175.

84. Ibid., 1869, p. 66.

85. Ibid., p.167.

86. Ibid., 1871, p. 275.

87. Ibid., 1870, p. 26.

88. Ibid., 1875, p. 131.

89. « Adénomes », Ibid., 1975, p. 165.

90. « Type scrofuleux ", ibid., 1876, p. 47.

91. « Anencéphalie », ibid., 1870, p. 32.

92. F. Villard, « Tératologie », ibid. 1871, p. 165.

93. La Rédaction, « Cas de tératologie », ibid., 1869, p. 103-104.

94. Voir Gérard Tilles et Estelle Lambert, op. cit., URL : https://www.biusante.parisdescartes.fr/ stlouis/fr/04-01.htm (conculté le 16 avril 2021).

95. L'Abeille médicale, 1876 (t. XXXIII), p. 335.

96. Ibid.

97. Charles Robin, « Cas de tératologie », op. cit., 1869, p. 113.

98. Ibid., 1976, planche VI.

99. Op. cit., p. 95.

100. Op. cit., 1874, planche XXII, p. 180.

101. Marie Barthélémy, « Félix Méheux, artiste dermatographe », Patrimoine en revue, APHP, $\mathrm{n}^{\circ} 14$, p. 6 .

102. Frédérique Calcagno-Tristant, op. cit., p. 93.

103. Montméja et Hardy, op. cit., p. II. 
104. Parue en 1869, la revue n'est pas arrêtée par les difficultés de la guerre mais, sur ce secteur spécialisé, la majorité des périodiques poursuivent leur publication. Ce n'est donc pas un indicateur valable pour trancher quant à la bonne santé du périodique.

105. Voir Gérard Tilles, "La création du Musée de l'hôpital Saint-Louis », Histoire des sciences médicales, t. XXVIII, $\mathrm{n}^{\circ} 4,1994$, p. 352.

106. Arthur de Montméja et Jules Rengade, « Avant-propos », Revue photographique des hôpitaux de Paris, 1869, n.p.

107. «Travaux des sociétés savantes », L'Abeille médicale, 25 janvier 1869, n4, p. 33.

108. Ibid., 1869, p. 26-27.

109. La revue mentionne rapidement cette décoration en première page, pourtant la base de données LEONORE indique que cette distinction n'est actée qu'en 1879. Il est plus intéressant de constater que cette distinction n'est pas en lien avec l'investissement photographique et/ou médical de Montméja.

110. Voir Sandrine Mercèdre, «Les secrets du château de Rouffillac », Sud-Ouest, 20 avril 2015, [en ligne], URL : https://www.sudouest.fr/2015/04/20/les-secrets-du-chateau-derouffillac-1897137-1768.php?nic. Voir aussi le site officiel du château: https:// www.chateauderouffillac.com/history/

111. Le Petit Journal, 9 mars 1876, p. 3. Le nom du défunt empoisonné est orthographié de façon très aléatoire à ce moment : selon les journaux, on trouvera Garrigue ou Guarrigue, avec ou sans « $\mathrm{s}$ » final.

112. Ibid..

113. Il publie un bref texte en 1869, traitant d'un cas rencontré dans «l'arrondissement de Sarlat » : Dr. Montméja père, « Développement incomplet d'un bras », ibid., 1869, p. 67.

114. Une simple recherche sur le site Retronews en atteste.

115. L'Abeille médicale, 3 janvier 1876, p. 5.

116. René Semelaigne, Les Pionniers de la psychiatrie française avant et après Pinel, vol. 2, Paris, J.-B. Baillière, 1932, p. 243.

117. Voir Georges Didi-Huberman, op. cit.

118. Albert Londe, La Photographie médicale: application aux sciences médicales et physiologiques, Paris, Gauthier-Villars, 1893, p. 1.

119. Ibid., p. 64.

120. Voir Monique Sicard, «La photographie scientifique, les académismes et les avant-gardes », Alliage, $\mathrm{n}^{\circ} 39$, juillet 1999, p. 67-78, en ligne : http://revel.unice.fr/alliage/index.html?id=3997.

\section{RÉSUMÉS}

La Revue photographique des hôpitaux de Paris est un objet trouble. S'attribuant le rôle de vitrine pour les hôpitaux de Paris, elle met en scène la rencontre de deux milieux diamétralement opposés : le public médical, éduqué et privilégié, et les cas pathologiques les plus exceptionnels $\mathrm{du}$ Paris populaire. S'inscrivant dans une modernité typique du Second Empire, où la centralisation et la spectacularisation des merveilles de la science et de l'industrie sont la règle, elle remplit plus ou moins consciemment trois fonctions : offrant un panorama saisissant de la vie du Paris populaire et de ses accidents biologiques, elle veut raconter des «histoires » de maladies sélectionnées pour leur rareté, en les accompagnant d'une iconographie qu'elle érige en 
argument de vente principal via son titre. Cette étude montre que faute de ligne éditoriale suffisamment développée, cette revue est d'une extrême ambiguïté.

The Revue photographique des hôpitaux de Paris stages the meeting of two paradoxical elites: the medical, educated and privileged public, and the most exceptional pathological cases of popular Paris. Sprung from the Second Empire, where centralization and spectacularization of the wonders of science and industry are the rule, this periodical fulfills - more or less consciously three functions: offering a striking panorama of the life of popular Paris and its biological accidents, the review wants to tell "stories" of diseases selected for their rarity, accompanying them with an iconography set up as the main selling point. Our study shows that, by its lack of editorial line, this review is extremely ambiguous.

\section{INDEX}

Mots-clés : presse médicale, photographie scientifique, Paris, hôpitaux, pathologie

Keywords : medical press, scientific photography, Paris, hospitals, pathology

\section{AUTEUR}

\section{AXEL HOHNSBEIN}

Université de Bordeaux, EA 4574 SPH 